\title{
Examination of echocardiographic parameters for the early diagnosis of cardiac dysfunction in beta thalassemia major patients
}

\author{
Aysen Turedi Yildirim ${ }^{1}$, Yesim Oymak ${ }^{2}$, Yontem Yaman $^{2}$, Timur Mese ${ }^{3}$, Selen \\ Bayraktaroglu $^{4}$, Yesim Aydinok ${ }^{5}$, Canan Vergin ${ }^{2}$
}

\section{Abstract}

Objective: This study aimed to determine the utility of tissue Doppler, pulse-wave Doppler, and conventional echocardiography for the detection of cardiac dysfunction in the early period in beta thalassemia patients.

Methods: Forty beta thalassemia major patients undergoing regular blood transfusion and chelation therapy and 30 healthy controls with similar demographic factors were included in the study. Both the patient and control groups were examined with, tissue Doppler, pulse-wave Doppler, motion-mode echocardiography. Ferritin values and $\mathrm{T} 2 *$ magnetic resonance measurements were recorded for those in the patient group.

Results: Diastolic dysfunction indicators like early ventricular filling rate, atrial contraction rate, mitral A wave time, and reverse pulmonary venous flow velocity were higher in the patient group $(p<0.001, p=0.003$, $\mathrm{p}<0.001, \mathrm{p}=0.006$, respectively), the deceleration time and pulmonary venous systolic flow velocity $(\mathrm{p}=0.035, \mathrm{p}=0.033$, respectively) were lower. Moreover, in thalassemia major patients, there were significant increases in the ratios of early ventricular filling rate to early diastolic myocardial rate in the lateral, basal and midseptal walls $(\mathrm{p}<0.001, \mathrm{p}=0.001, \mathrm{p}=0.012$, respectively). While the difference between the ferritin values and echocardiographic data was not significant in patient, it was significant between patients with severe and no cardiac iron load regarding an increase in left ventricular enddiastolic thickness and a decrease in left ventricular ejection fraction values $(\mathrm{p}=0.045, \mathrm{p}=0.049$, respectively).

Conclusions: In beta thalassemia major patients, tissue Doppler, pulse-wave Doppler and conventional echocardiography can be used to detect cardiac dysfunction in the early periods in cases where T2* magnetic resonance is not available.

Keywords: Beta thalassemia major, cardiac dysfunction, pulse-wave Doppler echocardiography, tissue Doppler echocardiography, conventional echocardiography, T2* magnetic resonance

\section{Introduction}

Thalassemia is a genetic disorder characterized by ineffective hematopoiesis, which iscaused by insufficient production of globin subunits and increased hemolysis. Patients with this disorder require frequent blood transfusions $(1,2)$, which can cause iron accumulation in the heart that can lead to severe cardiac complications

Despite the improvements in chelation therapy, cardiac complications remain the main cause of mortality and morbidity in thalassemia major patients (4). Several procedures have been used to detect the iron load in the heart. Echocardiography is a non-invasive, repeatable, and relatively inexpensive technique that is capable of the absolute diagnosis of heart diseases (5). Tissue Doppler imaging is a recently developed echocardiographic technique that can be used to obtain global and local measurements of myocardial systolic and diastolic velocities. Tissue Doppler echocardiography provides better definition of the left ventricular functions than does conventional echocardiography (6,7). Magnetic

Received: 10 Aug. 2014, Revised 20 Aug. 2014, Accepted 22 Aug. 2014, Available Online 30 Aug. 2014

${ }^{1}$ Department of Pediatric Hematology, Faculty of Medicine, Celal Bayar University, Manisa, Turkey

${ }^{2}$ Department of Pediatric Hematology, Dr. Behcet Uz Children's Hospital, Izmir, Turkey

${ }^{3}$ Department of Pediatric Cardiology, Dr. Behcet Uz Children's Hospital, Izmir, Turkey

${ }^{3}$ Department of Radiology, Faculty of Medicine, Ege University, Izmir, Turkey

${ }^{4}$ Department of Pediatric Hematology, Faculty of Medicine, Ege University, Izmir, Turkey

*Corresponding Author: Aysen Turedi Yildirim aysen_turedi@hotmail.com 


\section{Yildirim et al.}

resonance imaging (MRI) systems, which can detect myocardial iron load and myocardial functions, revolutionized the therapy of beta thalassemia major patients. An application known as $\mathrm{T} 2 *$ magnetic resonance is a powerful technique that detects the iron load by measuring myocardial relaxation time $(8) . \mathrm{T} 2 *$ magnetic resonance allows for the preclinical detection of myocardial iron load, the prospective categorization of the cardiac risks, and the observation of the response to changes in chelation therapy (9). However, its use in developing countries is limited because it is not cost-effective or easy to access.

In this study, we aimed to examine the efficiency of conventional echocardiography, tissue Doppler, and pulse-wave Doppler echocardiography, which are easy to access and economical compared to T2* magnetic resonance, for the detection of cardiac dysfunction at an early phase (i.e., before systolic dysfunction occurs) in beta thalassemia major patients.

\section{Materials and Methods}

Forty beta thalassemia major patients undergoing regular blood transfusion and chelation therapy (mean age: $12.8 \pm 3.34$ years) in Dr. Behcet Uz Children's Hospital and 30 healthy controls with similar demographic factors (e.g., age and sex) (mean age: $11.9 \pm 3.17$ ) were included in the study. Height, weight, systolic and diastolic blood pressure, heart beat rate and hemogram values were recorded in both the patient and control groups. The patients were categorized into three groups according to their ferritin values as follows: $<1000$ ng/dl, 1000-2500 ng/dl and >2500 ng/dl. Ethical approval was obtained from the hospital ethical commission and parental consent forms were collected.

Echocardiographic studies were performed at least 24 hours after blood transfusion. Motion mode, two dimensional imaging, tissue Doppler
Doi: $10.17546 / \mathrm{msd} .68956$

imaging and Doppler (pulse-wave, continuouswave) echocardiographic examinations were performed with a Vivid 3 (GE Healthcare, Milwaukee, WI) ultrasound system using a 3-5 $\mathrm{MHz}$ transducer on both the patient and control groups. Images were taken from the 3rd or 4th intercostal space while the patient was lying down or at a slight left lateral decubitus position. The measurements were recorded according to the American Society of Echocardiography guidelines (10).

Motion-mode echocardiography: The systolic functions of the left ventricle were evaluated using motion-mode echocardiography as follows: Left ventricle internal diameter at enddiastole, interventricular septum diastolic thickness, left ventricular posterior wall thickness at end diastole, left ventricular diastolic mass, left ventricular diastolic mass index, fractional shortening and left ventricular ejection fraction.

Pulse-wave Doppler echocardiography: Mitral valve flow rates and diastolic functions were evaluated via pulmonary pulse Doppler as follows: Early ventricular filling rate, atrial contraction rate, the ratio of early ventricular filling rate to contraction rate, mitral valve $\mathrm{A}$ wave time, deceleration time, pulmonary venous systolic flow velocity, pulmonary venous diastolic flow velocity and reverse pulmonary venous flow velocity. The ratios of pulmonary venous systolic flow velocity to pulmonary venous diastolic flow velocity were calculated.

Tissue Doppler echocardiography: Diastolic functions were evaluated by recording the myocardial signals as follows: Systolic myocardial velocity, early diastolic myocardial velocity, late diastolic myocardial velocity, pulmonary venous systolic flow velocity, pulmonary venous diastolic flow velocity, the ratio of early ventricular filling velocity to diastolic myocardial velocity, isovolumic relaxation time, and isovolumic 


\section{Yildirim et al.}

contraction time. Myocardial performance index was calculated. All measurements of the patient and control groups were performed by the same pediatric cardiologist who was blinded to the $\mathrm{T} 2 *$ magnetic resonance results.

T2* magnetic resonance: All cardiac magnetic resonance examinations were performed with a $1.5 \mathrm{~T}$ magnetic resonance (Symphony,
Doi: $10.17546 / \mathrm{msd} .68956$

with a $\mathrm{T} 2 *$ value $\leq 10 \mathrm{~ms}$ were considered to have severe iron accumulation.

Patients with left ventricular systolic dysfunction (Left ventricular ejection fraction $<\% 54$, fractional shortening $<\% 27$ ), congenital or acquired valve disease, hypertension, and patients aged younger than 8 years or older than 18 years were not included in the study.

Table 1: Clinical and demographic data of the patient and control groups.

\begin{tabular}{lccc}
\hline & $\begin{array}{c}\text { Study Group } \\
\text { (n: } 40)\end{array}$ & $\begin{array}{c}\text { Control Group } \\
\text { (n: } 30)\end{array}$ & p value \\
\hline Male/Female & $19 / 21$ & $13 / 17$ & 0.811 \\
Age $($ years) & $12.8 \pm 3.34$ & $11.9 \pm 3.17$ & 0.253 \\
Height $(\mathrm{cm})$ & $140.03 \pm 12.48$ & $145.43 \pm 15.24$ & 0.108 \\
Weight $(\mathrm{kg})$ & $35.76 \pm 9.53$ & $40.78 \pm 11.83$ & 0.054 \\
BMI $\left(\mathrm{kg} / \mathrm{m}^{2}\right)$ & $17.96 \pm 2.86$ & $18.89 \pm 2.87$ & 0.184 \\
Systolic tension $(\mathrm{mm} / \mathrm{Hg})$ & $114.55 \pm 9.46$ & $117.50 \pm 8.87$ & 0.189 \\
Diastolic tension $(\mathrm{mm} / \mathrm{Hg})$ & $68.22 \pm 8.38$ & $71.17 \pm 6.13$ & 0.109 \\
Heart beat $(/ \mathrm{min})$ & $98.33 \pm 11.79$ & $96.03 \pm 12.95$ & 0.443 \\
Hemoglobin $(\mathrm{gr} / \mathrm{dl})$ & $12.98 \pm 1.67$ & $12.39 \pm 1.23$ & 0.866 \\
\hline
\end{tabular}

Siemens, Erlanger, Germany). Myocardial iron load was evaluated by the myocardial $\mathrm{T} 2 *$ measurement. Images were obtained from the late diastolic phase of the cardiac cycle by electrocardiographic synchronization. The myocardial $\mathrm{T} 2 *$ measurement was performed using a multiecho technique when a cardiac event was triggered with a single-breathhold (FOV, 400 mm; TR,135; TE, 2.6-22.3 (8 echo sequence) with the following settings: flip angle, 20; section thickness, 10mm; matrix, 192x75, NEX,1; Band width (Hz/pixel)).All magnetic resonance analyses were conducted by a radiologist who was blinded to the echocardiography results. The T2* analysis was performed by Thalassemia tools (Thalassemia Tools, Cardiovascular Imaging Solutions, London, UK). The region of interest was selected on the interventricular septum. The signal intensity of this region was measured using a signal intensity-echo time curve (curve formula $=\mathrm{y}=\mathrm{Ke}^{-}$ $\left.{ }^{\mathrm{TE} / \mathrm{T} 2 *}\right)$. Cases with a $\mathrm{T} 2 *$ value $>20 \mathrm{~ms}$ were considered to have no iron accumulation, cases with a T2* value between 10 and $20 \mathrm{~ms}$ were accepted as having intermediate iron accumulation, and those

\section{Statistical Methods}

Statistical evaluations were performed using statistical tool SPSS 15.0 for Windows. A Chi-Square test or t-test was used to compare the characteristics of the two groups a Mann-Whitney $\mathrm{U}$ test was used to compare the echocardiographic parameters between the two groups. The ferritin and $\mathrm{T} 2 *$ values were compared to the echocardiographic parameters by a Kruskal-Wallis test.P values less than $0.05(\mathrm{p}<0.05)$ were accepted as statistically significant.

Results

The basic characteristic features of the beta thalassemia major patients and the healthy controls are summarized in Table 1. Conventional echocardiographic data including left ventricle internal diameter at end-diastole, inter ventricular septal diastolic thickness, left ventricular diastolic mass, and left ventricular diastolic mass index were higher in the patient group than in the controls $(\mathrm{p}=0.009, \mathrm{p}=0.009, \mathrm{p}=0.026, \mathrm{p}<0.001$, respectively) (Table 2). While diastolic dysfunction indicators 


\section{Yildirim et al.}

early ventricular filling rate, atrial contraction rate, mitral valve A wave duration and reverse pulmonary venous atrial velocity measured by pulse-wave Doppler echocardiography were higher in the patient group $(\mathrm{p}<0.001, \mathrm{p}=0.003, \mathrm{p}<0.001$, $\mathrm{p}=0.006$, respectively), deceleration time and pulmonary venous systolic flow velocity values were lower $(p=0.035, \quad p=0.033, \quad p<0.001$, respectively) (Table 3). Systolic myocardial velocity lateral, early diastolic myocardial velocity midseptal and the ratio of early ventricular flow velocity to early diastolic myocardial velocity ( lateral, basal and midseptal) data measured by tissue Doppler echocardiography were higher in the patient group $(p=0.031, p=0.019, p<0.001, p=0.001$, $\mathrm{p}=0.012$, respectively) while late diastolic myocardial velocity lateral, isovolumic contraction time lateral and isovolumic contraction time midseptal values were lower $(p=0.032, p=0.021$, $\mathrm{p}=0.011$, respectively) (Table 4).

The mean ferritin value of patients was $2595.46 \mathrm{ng} / \mathrm{dl}( \pm 1929.85)$. There were 10 patients with $<1000 \mathrm{ng} / \mathrm{dl}$ ferritin, 14 patients with 1000 $2500 \mathrm{ng} / \mathrm{dl}$ ferritin and 16 patients with $>2500 \mathrm{ng} / \mathrm{dl}$ ferritin. There were no significant correlations between the ferritin values and the conventional, pulse-wave Doppler or tissue Doppler echocardiographic data. The mean cardiac $\mathrm{T} 2 *$ value was $23.13 \mathrm{~ms}( \pm 10.18)$. There were 26 patients with no cardiac iron load (T2*>20 ms), nine patients with intermediate iron load $(\mathrm{T} 2 *=10$ $20 \mathrm{~ms}$ ) and five patients with severe cardiac iron load $(\mathrm{T} 2 *<10 \mathrm{~ms})$. There was no correlation
Doi: $10.17546 / \mathrm{msd} .68956$

between the $\mathrm{T} 2 *$ values and the PW Doppler or tissue Doppler echocardiography data $(\mathrm{p}>0.05)$. There was a statistically significant difference between patients with severe cardiac iron load and those with no iron load with respect to the increase in left ventricle internal diameter at end-diastole values and the decrease in left ventricular ejection fraction values detected by conventional echocardiography $(p=0.045$ and $p=0.049$, respectively).

\section{Discussion}

One of the main causes of mortality and morbidity in beta thalassemia major patients is iron accumulation due to blood transfusions (4). Therefore, the ability to track cardiac dysfunction and cardiac iron load in these patients is vital. The early ventricular filling rate value measured by tissue Doppler echocardiography is an indicator of ventricular relaxation and diastolic function (11). Spirito et al. examined diastolic abnormalities in 32 beta thalassemia major patients with tissue Doppler echocardiography and found that the increase in early ventricular filling rate value was significant as was the decrease in deceleration time value (12). In our study, we also reported that the early ventricular filling rate values of the patients were significantly higher than those of the controls (1.21 $\pm 0.1 \mathrm{~m} / \mathrm{s}, 0.99 \pm 0.15 \mathrm{~m} / \mathrm{s}, \mathrm{p}<0.001$, respectively) and that the deceleration time values of the patients were significantly lower than those of the controls $(157.05 \pm 39.1$ vs $170.57 \pm 34.93 \mathrm{p}=0.035$, respectively). 
Table 2: Conventional echocardiographic data of the patient and control groups.

\begin{tabular}{lccc}
\hline & $\begin{array}{c}\text { Study Group } \\
(\mathrm{n}: 40)\end{array}$ & $\begin{array}{c}\text { Control Group } \\
(\mathrm{n}: 30)\end{array}$ & $\mathrm{p}$ value \\
\hline LVIDd $(\mathrm{cm})$ & $4.06 \pm 0.43$ & $3.76 \pm 0.46$ & $\mathbf{0 . 0 0 9}$ \\
IVSd $(\mathrm{cm})$ & $0.86 \pm 0.16$ & $0.77 \pm 0.09$ & $\mathbf{0 . 0 0 9}$ \\
LVPWd(cm) & $0.82 \pm 0.14$ & $0.84 \pm 0.15$ & 0.494 \\
LVd Mass $(\mathrm{gr})$ & $106.46 \pm 32.29$ & $88.58 \pm 27.56$ & $\mathbf{0 . 0 2 6}$ \\
LVd MI $(\mathrm{gr} / \mathrm{m} 2)$ & $92.00 \pm 24.78$ & $69.31 \pm 16.97$ & $<\mathbf{0 . 0 0 1}$ \\
FS $(\%)$ & $36.31 \pm 5.81$ & $37.50 \pm 4.60$ & 0.427 \\
EF(\%) & $66.17 \pm 7.61$ & $68.08 \pm 5.68$ & 0.373 \\
\hline
\end{tabular}

LVIDd: Left ventricle internal diameter at end-diastole, IVSd: Inter ventricular septal diastolic thickness, LVPWd: Left ventricular posterior wall thickness at end diastole, LVd Mass: Left ventricular diastolic mass, LVd MI: Left ventricular diastolic mass index, FS: Fractional shortening, EF: Left ventricular ejection fraction

Deceleration time abnormality
accompanying early ventricular filling rate was considered to be an indicator of diastolic dysfunction. Parale et al. evaluated the left ventricular functions by pulse-wave Doppler echocardiography in beta thalassemia major patients and reported a significant increase in early ventricular filling rate / early diastolic myocardial velocity value and a significant extension of isovolumic relaxation time (13). In our study, we found that the early ventricular filling rate / early diastolic myocardial velocity measured from the lateral, basal and midseptal walls in beta and a significant decrease in late diastolic myocardial velocity (lateral) as measured by tissue Doppler echocardiography in beta thalassemia major patients as compared to the controls ( $p=0.031, p=0.019, p=0.032$, respectively).

The pulmonary venous flows, which are other indicators of diastolic function,were also measured in all groups. The disruption of ventricular relaxation might increase the atrial load, which in turn reduces the left atrial compliance and disrupts atrial relaxation. As a result, pulmonary venous systolic flow velocity often decreases while pulmonary venous diastolic flow velocity and

Table 3: Pulse-wave Doppler echocardiographic data in the patient and control groups.

\begin{tabular}{lccc}
\hline & $\begin{array}{c}\text { Study Group } \\
(\mathrm{n}: 40)\end{array}$ & $\begin{array}{c}\text { Control Group } \\
(\mathrm{n}: 30)\end{array}$ & $\mathrm{p}$ value \\
\hline $\mathrm{E}(\mathrm{m} / \mathrm{s})$ & $1.21 \pm 0.1$ & $0.99 \pm 0.15$ & $<\mathbf{0 . 0 0 1}$ \\
$\mathrm{A}(\mathrm{m} / \mathrm{s})$ & $0.76 \pm 0.37$ & $0.62 \pm 0.11$ & $\mathbf{0 . 0 0 3}$ \\
E/A & $1.73 \pm 0.42$ & $1.61 \pm 0.29$ & 0.206 \\
MVA $(\mathrm{ms})$ & $129.23 \pm 34.94$ & $101.89 \pm 38.74$ & $<\mathbf{0 . 0 0 1}$ \\
DT $(\mathrm{ms})$ & $157.05 \pm 39.1$ & $170.57 \pm 34.93$ & $\mathbf{0 . 0 3 5}$ \\
PVS $(\mathrm{ms})$ & $0.66 \pm 0.17$ & $0.77 \pm 0.17$ & $\mathbf{0 . 0 3 3}$ \\
PVD $(\mathrm{ms})$ & $0.63 \pm 0.17$ & $0.67 \pm 0.16$ & 0.805 \\
PVS/PVD & $1.08 \pm 0.42$ & $1.17 \pm 0.25$ & 0.233 \\
PVAR (m/s) & $0.45 \pm 0.13$ & $0.39 \pm 0.06$ & $\mathbf{0 . 0 0 6}$ \\
\hline
\end{tabular}

E: Early ventricular filling rate, A: Atrial contraction rate, E/A: The ratio of early ventricular filling rate to atrial contraction rate, MVA: Mitral valve A wave duration, DT: Deceleration time, PVS: Pulmonary venous systolic flow velocity, PVD: Pulmonary venous diastolic flow velocity, PVAR: Reverse pulmonary venous atrial velocity.

thalassemia major patients were significantly higher than those of the controls $(\mathrm{p}<0.001, \mathrm{p}=0.001$, $\mathrm{p}=0.012$, respectively). Also, there was a significant increase in systolic myocardial velocity (lateral) and early diastolic myocardial velocity (midseptal) reverse pulmonary venous atrial velocity wave amplitude and duration increase (14). In a study by Kremastinos et al.,early ventricular filling rate and atrial contraction rate values as well as pulmonary venous systolic flow velocity and pulmonary 


\section{Yildirim et al.}

venous diastolic flow velocity flow velocities were higher in beta thalassemia major patients, while there were no significant differences in the ratio of early ventricular filling rate to atrial contraction rate, deceleration time and isovolumic relaxation time values between patients and the control group (15). In our study, we found that the pulmonary venous systolic flow velocity values were lower in beta thalassemia patients when compared to the control group $(0.66 \pm 0.17 \mathrm{~ms}$ vs $0.77 \pm 0.17 \mathrm{~ms}$,
Doi: $10.17546 / \mathrm{msd} .68956$

fraction value (16). Ucar et al. reported an evident increase in myocardial performance index as measured by tissue Doppler echocardiography in 36 beta thalassemia patients with normal conventional echocardiographic parameters when compared to the control group (17). In our study, we could not detect a significant difference between the two groups with regards to the myocardial performance index as measured by tissue Doppler echocardiography.

Table 4: Tissue Doppler echocardiographic data in the patient and control groups.

\begin{tabular}{lccc}
\hline & $\begin{array}{c}\text { Study Group } \\
\text { (n: } 40)\end{array}$ & $\begin{array}{c}\text { Control Group } \\
\text { (n: } 30)\end{array}$ & p value \\
\hline Sm- lateral (ms) & $0.11 \pm 0.12$ & $0.10 \pm 0.02$ & $\mathbf{0 . 0 3 1}$ \\
Sm- basal (ms) & $0.08 \pm 0.01$ & $0.09 \pm 0.02$ & 0.438 \\
Sm- midseptal (ms) & $0.07 \pm 0.01$ & $0.09 \pm 0.15$ & 0.455 \\
Em- lateral (ms) & $0.19 \pm 0.02$ & $0.19 \pm 0.02$ & 0.609 \\
Em- basal (ms) & $0.16 \pm 0.09$ & $0.15 \pm 0.02$ & 0.992 \\
Em- midseptal (ms) & $0.13 \pm 0.02$ & $0.12 \pm 0.01$ & $\mathbf{0 . 0 1 9}$ \\
Am- lateral (ms) & $0.07 \pm 0.02$ & $0.08 \pm 0.01$ & $\mathbf{0 . 0 3 2}$ \\
Am- basal (ms) & $0.06 \pm 0.01$ & $0.06 \pm 0.01$ & 0.531 \\
Am-midseptal (ms) & $0.06 \pm 0.01$ & $0.05 \pm 0.01$ & 0.481 \\
IVRT- lateral (ms) & $56.49 \pm 7.57$ & $58.42 \pm 8.81$ & 0.549 \\
IVRT- basal (ms) & $56.00 \pm 8.33$ & $57.59 \pm 8.27$ & 0.288 \\
IVRT-midseptal (ms) & $54.23 \pm 6.22$ & $56.76 \pm 7.16$ & 0.077 \\
IVCT- lateral (ms) & $62.64 \pm 9.11$ & $66.64 \pm 7.78$ & $\mathbf{0 . 0 2 1}$ \\
IVCT- basal (ms) & $60.85 \pm 11.78$ & $66.23 \pm 9.55$ & 0.146 \\
IVCT-midseptal (ms) & $60.32 \pm 10.69$ & $66.03 \pm 8.15$ & $\mathbf{0 . 0 1 1}$ \\
E/Em- lateral & $6.37 \pm 1.07$ & $5.21 \pm 0.69$ & $<\mathbf{0 . 0 0 1}$ \\
E/Em- basal & $8.34 \pm 1.21$ & $6.65 \pm 1.36$ & $\mathbf{0 . 0 0 1}$ \\
E/Em- midseptal & $8.99 \pm 1.62$ & $8.07 \pm 1.35$ & $\mathbf{0 . 0 1 2}$ \\
MPI & $0.46 \pm 0.04$ & $0.47 \pm 0.05$ & 0.772 \\
\hline
\end{tabular}

Sm: Systolic myocardial velocity, Em: Early diastolic myocardial velocity, Am: Late diastolic myocardial velocity, PVS: Pulmonary venous systolic flow velocity, PVD: Pulmonary venous diastolic flow velocity, $\mathrm{E} / \mathrm{E}_{\mathrm{m}}$ :The ratio of early ventricular flow velocity to early diastolic myocardial velocity, IVRT: Isovolumic relaxation time, IVCT: Isovolumic contraction time, MPI: Myocardial performance index.

$\mathrm{p}=0.033$ ) while there were no differences in pulmonary venous diastolic flow velocity values. There was a significant increase in reverse pulmonary venous atrial velocity values $(0.45 \pm$ $0.13 \mathrm{~m} / \mathrm{s}$ vs0.39 $\pm 0.06 \mathrm{~m} / \mathrm{s}, \mathrm{p}=0.006)$ in then patient group when compared to the controls.

Myocardial performance index is a

Doppler index that enables the combined evaluation of left ventricular systolic and diastolic functions. It is a numerical value derived by dividing the sum of the isovolumic contraction time and isovolumic relaxation time by the left ventricular ejection
Conventional echocardiography is an economical, practical and frequently used technique for the evaluation of heart diseases (18). Stakos et al. found that the left ventricular mass and expansion in the left atrium as measured by conventional echocardiography was significantly increased in beta thalassemia major patients as compared to a control group, although there was no increase in cardiac iron load (19). In our study, left ventricle internal diameter at end-diastole, inter ventricular septal diastolic thickness, left ventricular diastolic mass and left ventricular 


\section{Yildirim et al.}

diastolic mass index values as measured by conventional echocardiography were significantly higher in the patient group than in the control group $(\mathrm{p}=0.009, \quad \mathrm{p}=0.009, \quad \mathrm{p}=0.026, \quad \mathrm{p}<0.001$, respectively).

While the serum ferritin level has a limited prognostic value for reflecting iron stores in iron overloaded patients, it is still valuable as an indirect indicator of iron stores. Moreover, it is a widely used and cost-effective method (20). Silvilairat et al. examined the relationship between the serum ferritin values and tissue Doppler echocardiography parameters in 31 thalassemia patients with normal left ventricular functions. High ferritin levels $(>5000 \mathrm{ng} / \mathrm{ml})$ were correlated with a decrease in deceleration time values and an increase in the ratio of early ventricular flow velocity to early diastolic myocardial velocity values (7). In a study examining the diastolic index and ferritin values of 29 thalassemia patients with normal systolic functions, Ashena et al. reported an increase in the early ventricular filling rate and atrial contraction rate values of patients $>15$ years old compared to patients $<15$ years old (21). In our study, the early ventricular filling rate and atrial contraction rate values were significantly higher in the patient group when compared to the controls $(1.21 \pm 0.1 \mathrm{vs} 0.99 \pm$ $0.15 \mathrm{p}<0.001,0.76 \pm 0.37$ vs0.62 $\pm 0.11 \mathrm{p}=0.00$, respectively). The studies of Ashena et al and Parale et al. reported no correlation between ferritin values and diastolic indexes. In accordance with these recent studies, we also did not find a correlation between serum ferritin levels and diastolic dysfunction indexes as measured by both pulse-wave Doppler and tissue Doppler echocardiography, or with systolic dysfunction indexes as measured by conventional echocardiography. In regards to the correlation between body iron load and cardiac iron accumulation, it has been reported that it is still possible to observe cardiac iron accumulation
Doi: $10.17546 / \mathrm{msd} .68956$

despite low ferritin levels and the accumulation of iron in the liver (22). This may explain the lack of correlation between serum ferritin levels and diastolic dysfunction indexes.

Currently, cardiac MRI is the gold standard for myocardial tissue analysis in various cardiac diseases. However, its use in developing countries is limited due to its high cost and restricted access (8). In a prospective observational study of thalassemia major patients, it was demonstrated that patients with increased cardiac iron load $(\mathrm{T} 2 *<10 \mathrm{~ms}) \quad$ carry a high risk of cardiac insufficiency (23). In a study conducted in patients with a mean age of $18 \pm 6$ years (6-31 years), Aypar et al. examined the correlation of tissue Doppler echocardiography parameters with $\mathrm{T} 2 *$ magnetic resonance values. They found that the regional myocardial dysfunction was more evident in patients with $\mathrm{T} 2 * \leq 20 \mathrm{~ms}$ ( $86 \%$ of the patients). In addition, there was a significant correlation between midseptal Sm and Em values and cardiac iron load [24].

Vogel et al. evaluated 52 thalassemia major patients with the mean age of 29.2 years (1443 years) with tissue Doppler echocardiography and cardiac magnetic resonance and found a significant correlation between a cardiac iron load of $\mathrm{T} 2 *<20 \mathrm{~m}(73 \%$ of the patients) and myocardial rates (early diastolic, atrial and systolic myocardial velocities), which are diastolic dysfunction indicators [25]. In our study, we did not detect a significant correlation between $\mathrm{T} 2 *$ values and pulse-wave Doppler and tissue Doppler echocardiography indexes. This might be due to the lower mean age (12.8 \pm 3.3 years) of our patients compared to those of the previous studies and the low number of patients with $\mathrm{T} 2 *$ magnetic resonance values $<20 \mathrm{~ms}$ (35\% of the patients). Nonetheless, there was a weak correlation between the decrease in left ventricular ejection fraction value and the decrease in $\mathrm{T} 2 *$ value and between 


\section{Yildirim et al.}

the increase in left ventricular diastolic thickness value and the decrease in $\mathrm{T} 2 *$ value $(\mathrm{p}=0.049$, $\mathrm{p}=0.045$, respectively). Left ventricular ejection fraction in these three groups $(\mathrm{T} 2 *>20 \mathrm{~ms}, \mathrm{~T} 2 *=10$ $20 \mathrm{~ms}, \mathrm{~T} 2 *<10 \mathrm{~ms}$ ) were $68.00 \%, 63.08 \%$, and $61.82 \%$, respectively, and left ventricular posterior wall thickness at end diastole values were $4.03 \pm$ $0.39 \mathrm{~cm}, 4.14 \pm 0.41 \mathrm{~cm}$, and $4.45 \pm 0.03 \mathrm{~cm}$, respectively.

In conclusion, the ratio of early ventricular flow velocity to early diastolic myocardial velocity (lateral, basal and midseptal) values as measured by tisse Doppler echocardiography, early ventricular filling rate, atrial contraction rate, mitral valve $\mathrm{A}$ wave duration, reverse pulmonary venous atrial velocity, deceleration time, and pulmonary venous systolic flow velocity values as measured by pulse-wave Doppler echocardiography, and left ventricle internal diameter at end-diastole, inter ventricular septal diastolic thickness, left ventricular diastolic mass, and left ventricular diastolic mass index values as measured by conventional echocardiography show distinct differences between beta thalassemia major patients and healthy controls. In these patients, tissue Doppler, pulse-wave Doppler and conventional echocardiography could be used as economical and easily-accessible alternatives for the observation of cardiac dysfunction due to iron load where T2* magnetic resonance is not available.

\section{Acknowledgements: None.}

Financial Support: This research received no specific grant from any funding agency, commercial or not-for-profit sectors

Conflict of Interest: The authors declared that they had no conflicts of interest.
Doi: $10.17546 / \mathrm{msd} .68956$

\section{References}

[1] Lanzkowsky P, Atlas M. Hemolytic Anemia; Thalassemias. Manual of Pediatric Hematology and Oncology 4th ed. New York. 2005;181-191.

[2] Olivieri NF. The beta-thalassemias. The New England journal of medicine. 1999;341(2):99-109.

[3] Kyriacou K, Michaelides Y, Senkus R, Simamonian K, Pavlides N, Antoniades L, et al. Ultrastructural pathology of the heart in patients with beta-thalassaemia major. Ultrastructural pathology. 2000;24(2):75-81.

[4] Modell B, Khan M, Darlison M. Survival in betathalassaemia major in the UK: data from the UK Thalassaemia Register. Lancet. 2000;355(9220):2051-2.

[5] Kolnagou A, Economides C, Eracleous E, Kontoghiorghes GJ. Low serum ferritin levels are misleading for detecting cardiac iron overload and increase the risk of cardiomyopathy in thalassemia patients. The importance of cardiac iron overload monitoring using magnetic resonance imaging T2 and T2*. Hemoglobin. 2006;30(2):219-27.

[6] Isaaz K, Thompson A, Ethevenot G, Cloez JL, Brembilla B, Pernot C. Doppler echocardiographic measurement of low velocity motion of the left ventricular posterior wall. The American journal of cardiology. 1989;64(1):66-75.

[7] Silvilairat S, Sittiwangkul R, Pongprot Y, Charoenkwan P, Phornphutkul C. Tissue Doppler echocardiography reliably reflects severity of iron overload in pediatric patients with beta thalassemia. European journal of echocardiography : the journal of the Working Group on Echocardiography of the European Society of Cardiology. 2008;9(3):368-72.

[8] Pennell DJ. T2* magnetic resonance and myocardial iron in thalassemia. Annals of the New York Academy of Sciences. 2005;1054:373-8.

[9] Wood JC, Noetzli L. Cardiovascular MRI in thalassemia major. Annals of the New York Academy of Sciences. 2010;1202:173-9.

[10] Quinones MA, Otto CM, Stoddard M, Waggoner A, Zoghbi WA, Doppler Quantification Task Force of the N, et al. Recommendations for quantification of Doppler echocardiography: a report from the Doppler Quantification Task Force of the Nomenclature and Standards Committee of the American Society of Echocardiography. Journal of the American Society of Echocardiography : official publication of the American Society of Echocardiography. 2002;15(2):167-84.

[11] Hirota Y. A clinical study of left ventricular relaxation. Circulation. 1980;62(4):756-63.

[12] Spirito P, Lupi G, Melevendi C, Vecchio C. Restrictive diastolic abnormalities identified by Doppler echocardiography in patients with thalassemia major. Circulation. 1990;82(1):88-94

[13] Parale GP, Pawar SS, Tapare VS. Assessment of LV diastolic function in patients with beta-thalassemia major with special reference to E/Eann ratio. Journal of pediatric hematology/oncology. 2009;31(1):69-73. 


\section{Yildirim et al.}

[14] Ho CY. Echocardiographic Assessment of Diastolic Function. In: Solomon SD editor. Essential Echocardiography: A Practical Handbook with DVD. New Jersey: Humana Press Inc. 2007:119-131.

[15] Kremastinos DT, Tsiapras DP, Tsetsos GA, Rentoukas EI, Vretou HP, Toutouzas PK. Left ventricular diastolic Doppler characteristics in beta-thalassemia major. Circulation. 1993;88(3):1127-35

[16] Tei C, Ling LH, Hodge DO, Bailey KR, Oh JK, Rodeheffer RJ, et al. New index of combined systolic and diastolic myocardial performance: a simple and reproducible measure of cardiac function--a study in normals and dilated cardiomyopathy. Journal of cardiology. 1995;26(6):357-66.

[17] Ucar T, Ileri T, Atalay S, Uysal Z, Tutar E, Ertem M. Early detection of myocardial dysfunction in children with beta-thalassaemia major. The international journal of cardiovascular imaging. 2009;25(4):379-86.

[18] Feigenbaum H. Role of M-mode technique in today's echocardiography. Journal of the American Society of Echocardiography : official publication of the American Society of Echocardiography. 2010;23(3):240-57; 335-7.

[19] Stakos DA, Margaritis D, Tziakas DN, Kotsianidis I, Chalikias GK, Tsatalas $\mathrm{K}$, et al. Cardiovascular involvement in patients with beta-thalassemia major without cardiac iron overload. International journal of cardiology. 2009;134(2):207-11
Doi: $10.17546 / \mathrm{msd} .68956$

[20] Tokgöz H, Çalıșkan Ü. Talasemi demir regülasyonu, metabolizması ve demir yükünün tayini. Türkiye Klinikleri J Hem Onc-Special Topics. 2010;3:70-75.

[21] Ashena Z, Ghafurian S, Ehsani MA. The relation between left ventricular diastolic indices and serum ferritin in thalassemia major. Pediatric hematology and oncology. 2007;24(1):3-14.

[22] Fischer R, Harmatz PR. Non-invasive assessment of tissue iron overload. Hematology / the Education Program of the American Society of Hematology American Society of Hematology Education Program. 2009:215-21.

[23] Kirk P, Roughton M, Porter JB, Walker JM, Tanner MA, Patel J, et al. Cardiac T2* magnetic resonance for prediction of cardiac complications in thalassemia major. Circulation. 2009;120(20):1961-8.

[24] Aypar E, Alehan D, Hazirolan T, Gumruk F. The efficacy of tissue Doppler imaging in predicting myocardial iron load in patients with beta-thalassemia major: correlation with $\mathrm{T}_{2} *$ cardiovascular magnetic resonance. The international journal of cardiovascular imaging. 2010;26(4):413-21.

[25] Vogel M, Anderson LJ, Holden S, Deanfield JE, Pennell DJ, Walker JM. Tissue Doppler echocardiography in patients with thalassaemia detects early myocardial dysfunction related to myocardial iron overload. European heart journal. 2003;24(1):113-9. 